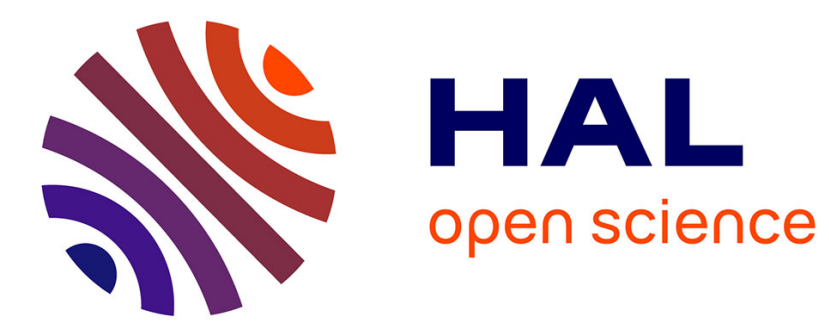

\title{
Computing the Fukui Function in Solid-State Chemistry: Application to Alkaline Earth Oxides Bulk and Surfaces
}

\author{
M. L Cerón, T. Gomez, M. Calatayud, C. Cárdenas
}

\section{To cite this version:}

M. L Cerón, T. Gomez, M. Calatayud, C. Cárdenas. Computing the Fukui Function in Solid-State Chemistry: Application to Alkaline Earth Oxides Bulk and Surfaces. Journal of Physical Chemistry A, 2020, 124 (14), pp.2826-2833. 10.1021/acs.jpca.0c00950 . hal-02873951

\section{HAL Id: hal-02873951 https://hal.science/hal-02873951}

Submitted on 18 Jun 2020

HAL is a multi-disciplinary open access archive for the deposit and dissemination of scientific research documents, whether they are published or not. The documents may come from teaching and research institutions in France or abroad, or from public or private research centers.
L'archive ouverte pluridisciplinaire HAL, est destinée au dépôt et à la diffusion de documents scientifiques de niveau recherche, publiés ou non, émanant des établissements d'enseignement et de recherche français ou étrangers, des laboratoires publics ou privés. 


\title{
Computing the Fukui Function in Solid-State
} Chemistry: Application to Alkaline Earth Oxides Bulk and Surfaces

\author{
M. L. Cerón, ${ }^{\dagger}$ T. Gomez, ${ }^{\ddagger}$ M. Calatayud, ${ }^{*}, \mathbb{q}$ and C. Cárdenas ${ }^{*}, \S, \|$ \\ $†$ Facultad de Ingeniería, Universidad Finis Terrae, Av. Pedro de Valdivia 1509, \\ Providencia, Santiago, Chile \\ $\ddagger$ Theoretical and Computational Chemistry Center, Institute of Applied Chemical Sciences, \\ Faculty of Engineering, Universidad Autonoma de Chile, El Llano Subercaceaux 2801, \\ Santiago, Chile \\ ๑Sorbonne Université, CNRS, Laboratoire de Chimie Théorique, LCT, F. 75005 Paris, \\ France \\ $\S$ Departamento de Física, Facultad de Ciencias, Universidad de Chile. Casilla 635, \\ Santiago, Chile \\ \|Centro para el Desarrollo de la Nanociencia y la Nanotecnología (CEDENNA), Avda. \\ Ecuador 3493, Santiago 9170124, Chile \\ E-mail: calatayu@lct.jussieu.fr; cardena@uchile.cl \\ Phone: +33 (0)1 44272505; +56 (2) 229787161. Fax: +33 (0)1 44274117
}

\begin{abstract}
Fukui functions (FF) are chemical descriptors useful to explain the reactivity of systems towards electron transfer. Whereas they have been widely employed for molecules, their application to extended systems is scarce. One of the reasons for the limited devel-
\end{abstract}


opment of such analysis in solids is the improper evaluation of FF in the usual computational approaches based on density functional theory and periodic boundary conditions. In this work we compare the available approaches and propose a new method, based on the interpolation of partially charged systems, that mitigates some of the problems encountered. We discuss the reactivity of alkaline earth oxides $(\mathrm{MgO}, \mathrm{CaO}, \mathrm{SrO}$ and $\mathrm{BaO}$ ) in terms of the FF analysis, providing a robust way to account for the higher reactivity of surface oxygen sites compared to bulk sites.

\section{Introduction}

Both, in organic and inorganic chemistry it is customary to classify reactions as being controlled by either electron-transfer or electrostatic effects. These are, however, two limiting cases as in actual reactions both effects are always present. This was first pointed out, in the context of molecular orbital theory, in seminal works by Klopman and Salem. ${ }^{1,2}$ Nevertheless, reactions mostly controlled by electron-transfer are of utmost importance to chemistry because most reactions where covalent bonds are broken and created fall within this category. It was Fukui who first realized that the reactivity of a molecule participating in an electron-transfer reaction is dictated by the density of HOMO or LUMO orbitals, depending on whether the molecule donates or accepts electrons. That simple idea is the basis of the frontier molecular orbital theory (FMO). ${ }^{3}$ The above-mentioned works by Salem and Klopman provided theoretical basis for the FMO theory by using molecular-orbital perturbation theory with single determinant wavefunctions. That is, FMO theory seems to be linked to a coarse approximation of the wavefunction. However, a strict generalization of Fukui's ideas is possible within the mathematical framework of the density functional theory (DFT). In 1978 Robert Parr realized that the Lagrange multiplier of the Euler-Lagrange equation of the DFT is nothing but the electronic chemical potential, ${ }^{4} \mu$, of the molecule and that its negative provides a non-empirical scale of electronegativity. This pioneering work was the first step to construct an unified corpus of chemical reactivity using DFT. Nowadays, this 
area of research is known by the name given by professor Paul Geerlings: conceptual DFT. Among the several reviews in the literature, ${ }^{5-9}$ the one made by Geerlings et al. in 2003 is still the most comprehensive one. ${ }^{10}$

The chemical potential of a molecule is unique in the sense that it is the same for all parts of the molecule. Therefore, if a reaction is to happen, the chemical potential has to change. Such change comes either from the flow of electrons, or from the change in the external potential of the nuclei on the electrons. If a molecule is perturbed in its number of electrons, $N$, and its external potential, $v(r)$, the first-order change of the chemical potential is

$$
d \mu=\left(\frac{\partial \mu}{\partial N}\right)_{v(r)} d N+\int\left(\frac{\delta \mu}{\delta v(\vec{r})}\right)_{N} \delta v(\vec{r}) d \vec{r} .
$$

Hence, a molecule can be considered reactive when its chemical potential changes easily. Parr called this the " $d \mu$ big is good" rule; for which a recent proof is available. ${ }^{11-13}$ Therefore, for a non-degenerate ground state, ${ }^{14-17}$ a perturbation will be more effective in changing the chemical potential if such perturbation (a reagent) occurs in places where $\left(\frac{\delta \mu}{\delta v(\vec{r})}\right)_{N}$ is large. A Maxwell relation shows that this functional derivative is also equal to the response of the electron density to changes in the number of electrons,

$$
f(\vec{r}) \equiv\left(\frac{\delta \mu}{\delta v(\vec{r})}\right)_{N}=\left(\frac{\partial \rho(\vec{r})}{\partial N}\right)_{v(\vec{r})}
$$

It is called the Fukui function because it generalizes the FMO theory. That is better seen when the Fukui functions are written in terms of the Kohn-Sham (KS) orbitals,

$$
f^{-/+}(\vec{r}) \equiv\left|\phi_{H O M O / L U M O}(\vec{r})\right|^{2}+\sum_{i=1}^{N}\left(\frac{\partial\left|\phi_{i}(\vec{r})\right|}{\partial N}\right)
$$

The Fukui function comprises two terms, the density of the HOMO (or LUMO) and a relaxation term. Note that there are two Fukui functions, one for donation of electrons 
(-,oxidation), and one for accepting electrons (+,reduction). It is so because of the discontinuity of the density as function of the number of electrons. ${ }^{18}$ In molecules, the relaxation term is usually very small because of the discrete nature of the KS orbitals' spectra. ${ }^{19}$ Summarizing, if electrostatic effects are ignored, a molecule is prone to donate/accept electrons in those sites where the Fukui function is large.

The Fukui function is extremely useful in characterizing the reactivity of molecules. ${ }^{20}$ Its use is so broadly accepted that it is discussed in modern organic chemistry books. However, its use in solid state chemistry is scarce because its calculation brings theoretical and computational challenges. Shortly, in molecules the density of the frontier orbitals is unambiguously defined, while in extended system the continuous character of the Bloch states makes difficult to identify just one frontier state, and the relaxation term could be large. In this work, we will discuss the advantages and problems of the methods available to compute the Fukui function in solid-state chemistry and present a new method that alleviates some of those difficulties. We compare three different ways of computing the Fukui function and analyze the family of alkaline earth metal oxides rocksalt MO structures ( $\mathrm{M}: \mathrm{Mg}, \mathrm{Ca}, \mathrm{Sr}$, Ba), both bulk and surface slab models.

\section{Computational details}

All calculations were done with the Vienna ab initio package VASP. ${ }^{21-23}$ DFT with periodic boundary conditions and plane wave basis set was used for computing the ground state of bulk and surfaces models. Core electrons were replaced by augmentation charges according to the projector-augmented wave (PAW) method. ${ }^{24}$ The GGA PBE exchange and correlation functional proposed by Perdew et al. was used. ${ }^{25}$ The plane wave basis set was truncated at $500 \mathrm{eV}$, which practically represents the complete basis set limit, for the bulk systems, and 400

$\mathrm{eV}$ for the slabs. For sampling the reciprocal space the Monkhorst-Pack ${ }^{26}$ scheme was chosen, using $25 \times 25 \times 25$ and $15 \times 15 \times 1$ k-meshes for bulk and surface slabs respectively. Integrals in 
the reciprocal space were done with the tetrahedron method with Blöchl corrections.

Bulk systems were computed using a conventional unit cell with 4 formula units. Properties such as optimized cell parameters, number of valence electrons, and band gap, are reported in supplementary material, Table S1. They agree with experimental and previous theoretical data obtained for similar computational settings. ${ }^{27-29}$ The (100) surfaces were cut from the optimized bulk structure, 6 oxide layers thick, and contain 12 formula units. The vacuum space between the surfaces was set to a minimum of $15 \AA$. Two sets of slabs were investigated: the asymmetric slab (the top 2 layers are allowed to relax) and the symmetric slab (the two top and two bottom layers were allowed to relax, the two central layers were kept fixed to the bulk positions). The conjugate-gradient algorithm was used in the geometry optimizations with a convergence threshold of $0.01 \mathrm{eV} / \AA$ for the forces. The effect of geometrical relaxation in the interlayer distance for the two slab models is reported in Table S2. A similar protocol was found to be able to describe properly the surface structure in alkaline earth oxides. ${ }^{28}$

The Fukui Function $f^{-}$condensed to atoms is computed by integrating the charge (CHGCAR file) into Voronoi volumes, using Henkelman's program. ${ }^{30}$ Only the oxygen values are reported.

\section{Results and Discussion}

In this section we present the computational schemes to compute the Fukui Function $f^{-}$, as well as an application to MO surfaces.

\section{Finite difference with $\Delta N= \pm q$}

Computing the Fukui function in finite systems is straightforward from a "finite difference" expression, 


$$
f^{-}(\vec{r})=\rho^{N}(\vec{r})-\rho^{N-1}(\vec{r})
$$

where $N$ stands for the system of interest and and N-1 for its vertical cation. A similar expression exists for $f^{+}$. Note that although Eq. 4 seems to be an approximation to the derivative of Eq.2, the difference in Eq. 4 is, in principle, exact. This exactness comes from the derivative discontinuity of the energy and the density as functions of the number of electrons. For finite systems, Perdew, Parr, and Baldus ${ }^{18}$ proved that density of an open system with $N_{0} \pm \delta q$ electrons, with $N_{0}$ an integer, is a linear combination of the densities of the systems with the closest integer number of electrons. Therefore, any departure from exactness form Eq. 4 comes from the approximations used to compute the density. In the special case of DFT, approximations to the exchange-correlation functional are designed to give good densities for integer number of electrons, but not for non-integer numbers. ${ }^{31}$

The discussion on how to compute the Fukui function has been so far given mostly for finite systems. ${ }^{32,33}$ We will now focus on alternatives on how to compute the Fukui function in extended systems with periodic boundary conditions (PBC). We will focus only on the Fukui function for removal of electrons $f^{-}$because in PBC calculations with GGA functional the conduction band is normally not as accurate as the valence band. However, similar results hold for $f^{+}$.

A natural extension of Eq.4 to extended systems suggests to compute the Fukui function by removing/adding electrons from/to the neutral system. However, this procedure leaves a charged cell or supercell, the images of which will interact electrostatically. Hence, adding or removing a whole electron can add a non-negligible unphysical external potential to the unitary cell. Naively, one may think that increasing the size of the unitary cell will fix this problem, but the Coulomb interaction is a long-range one and it does not matter how large the unit cell is, it never converges to zero. The magnitude of the effect of this electrostatic "noise" is not easy to determine a priory, but it is expected to be more significant in soft systems, such as metals with a large density of states (DOS) at the Fermi Level. Approximation 
for cancelling the electrostatic fields of charged images on the total energy are available, but these methods are usually limited to cubic unit cells. Some of us used this approach to identify the regions that maximize the Fukui function in $\mathrm{V}_{2} \mathrm{O}_{5}$ systems, allowing a qualitative visual inspection of the different oxygen surface sites, ${ }^{34}$ as well as a comparison of different vanadium-based systems.

In the present work we obtained the difference between the charge files for neutral and charged systems by adjusting the total number of electron per cell with the keyword NELECT in VASP. Table 1 displays the $f^{-}$values obtained for the four bulk systems with the finite differences procedure, for different values of charge, $\delta q$. It can be observed that the values are almost constant within each series: $\mathrm{MgO}$ (0.168), $\mathrm{CaO}$ (0.167-0.172), $\mathrm{SrO}(0.171-0.172)$ and $\mathrm{BaO}(0.157-0.160)$. There are not significant differences between the fractionl charges and the integer (charge -1 ) one. The $\mathrm{BaO}$ system exhibits always lower values for the $f^{-}$, 0.160, compared to $\mathrm{MgO}, \mathrm{CaO}$ and $\mathrm{SrO}$ that oscillate around 0.169 . The slightly lover value of $f^{-}$in $\mathrm{BaO}$ can be understood as a consequence of its larger softness and the condensation scheme. As $\mathrm{Ba}$ is the softest metal in the series, the frontier density is expected to be less localized around the oxygen atom. Hence, the Voronoi polyhedrons may be not an accurate representation of the space belonging to atoms.

Table 1: $f^{-}$obtained with finite differences (equation 4) for the four bulk systems of the alkaline earth metal oxide series

\begin{tabular}{lllll}
\hline$\delta q$ & $\mathrm{MgO}$ & $\mathrm{CaO}$ & $\mathrm{SrO}$ & $\mathrm{BaO}$ \\
\hline-0.05 & 0.168 & 0.167 & 0.171 & 0.157 \\
-0.10 & 0.168 & 0.169 & 0.172 & 0.157 \\
-0.15 & 0.168 & 0.171 & 0.172 & 0.157 \\
-0.20 & 0.168 & 0.172 & 0.172 & 0.157 \\
-0.25 & 0.168 & 0.171 & 0.172 & 0.157 \\
-0.30 & 0.168 & 0.172 & 0.171 & 0.157 \\
-1.00 & 0.168 & 0.169 & 0.171 & 0.160 \\
\hline
\end{tabular}




\section{Fukui functions from the local density of states}

An alternative to evaluate the Fukui function and that avoids calculations of charged cells is to compute the local softness from the local density of states (LDOS) $g(\vec{r}, E),[6,7]$ and from it to compute the Fukui function. The local softness is the derivative of the electron density with respect to the chemical potential, at constant external potential,

$$
s(\vec{r})=\left(\frac{\partial \rho(\vec{r})}{\partial \mu}\right)_{v(r)}=\left(\frac{\partial \rho(\vec{r})}{\partial N}\right)_{v(r)}\left(\frac{\partial N}{\partial \mu}\right)_{v(r)}=f(\vec{r}) S,
$$

That is, the Fukui function is equal to the local softness divided by the global softness, S. In systems with a fundamental gap, $\mathrm{S}$ is the inverse of the gap, while in gapless systems $\mathrm{S}$ is the DOS at the Fermi level. The link between the local softness and the LDOS is easily deduced from the definition of the density of the density in terms of the LDOS,

$$
\rho(\vec{r})=\int^{\mu} g(\vec{r}, E) d E,
$$

by taking the derivative with respect to the chemical potential (Fermi level)

$$
s(\vec{r})=g(\vec{r}, \mu)+\int^{\mu} \frac{\partial g(\mathbf{r}, E)}{\partial \mu} d E
$$

Eq. 7 is very similar to Eq. 3 in the sense that it has a frontier contribution, the LDOS at the Fermi level, and a relaxation term given by the integral. Contrary to molecules, the relaxation term could be not small because the density of states around the Fermi level is finite. That is, the states close to the Fermi level contribute to the Fukui function. Yet, there is not known analytical expression or method that allows to compute the relaxation term in Eq.7. A decade ago, Geerlings and one of us proposed and alternative expression to include the contribution of inner states to the local softness, ${ }^{28}$

$$
s(\vec{r})=\left(\frac{\partial \rho(\vec{r})}{\partial \mu}\right)_{v(r)}=\lim _{\delta \mu \rightarrow 0} \frac{1}{\delta \mu} \int_{\mu-\delta \mu}^{\mu} g(\vec{r}, E) d E,
$$


The reasoning behind this equation is that a derivative is nothing but a limit. Eq.8 has the advantage that the integral is very easy to evaluate and it is available in almost any solid state code. It has however two disadvantages: i) as the limit cannot be taken to zero, $s(r)$ depends on the value chosen for $\delta \mu$, and ii) it assumes that states below the Fermi level contribute as much as to $s(r)$ as the Fermi level. However, it is known from perturbation theory that the deeper the state, the smaller its contribution. Possible ways to weight the contribution of inner states have been discussed elsewhere. ${ }^{35-37}$

The Fukui function can be evaluated from Eq. 8 by normalizing it,

$$
f^{-}(\vec{r})=\frac{\lim _{\delta \mu \rightarrow 0} \int_{\mu-\delta \mu}^{\mu} g(\vec{r}, E) d E}{\lim _{\delta \mu \rightarrow 0} \int_{\mu-\delta \mu}^{\mu} g(\vec{r}, E) d \vec{r} d E} .
$$

We have applied this method to evaluate $f^{-}$for the bulk systems. The partial density of states between $\delta \mu$ and the Fermi level was obtained (from PARCHG files), and integrated in the Voronoi polyhedron. Table 2 shows the $f^{-}$values obtained normalized as in Eq. 9 for different values of $\delta \mu$. It can be observed that for a given oxide there is little dependence on $\delta \mu$. While $\mathrm{MgO}, \mathrm{CaO}$ and $\mathrm{SrO}$ show similar values around $0.23, \mathrm{BaO}$ exhibits slightly smaller values around 0.22. It is remarkable that the $f^{-}$computed with the LDOS has the same trend as $f^{-}$obtained by finite differences (Table 1). The fact that the values of $f^{-}$ are smaller for finite differences than for LDOS can be attributed to fact that Eq. 9 misses a part of the relaxation term of the exact expression of the softness (Eq. 7). Note that $\delta \mu$ and $\delta q$ do not represent the same quantity.

\section{Fukui functions from interpolated non-integer number of electrons.}

Using calculations with different number of electrons easily allows to incorporate relaxation

effects but it has the trouble of creating a fictitious electric field in systems with PBC. The approach based on the LDOS solves that problem but relaxation effects are not strictly incorporated. Another alternative is to use finite differences with fractional number of electrons. 
Table 2: $f^{-}$obtained with the DOS for the four bulk systems of the alkaline earth metal oxide series

\begin{tabular}{lllll}
\hline$\delta \mu$ & $\mathrm{MgO}$ & $\mathrm{CaO}$ & $\mathrm{SrO}$ & $\mathrm{BaO}$ \\
\hline-0.05 & 0.236 & 0.241 & 0.239 & 0.219 \\
-0.10 & 0.235 & 0.240 & 0.239 & 0.219 \\
-0.15 & 0.235 & 0.239 & 0.236 & 0.220 \\
-0.20 & 0.235 & 0.238 & 0.234 & 0.221 \\
-0.25 & 0.234 & 0.236 & 0.232 & 0.222 \\
-0.30 & 0.234 & 0.236 & 0.232 & 0.222 \\
-1.00 & 0.228 & 0.225 & 0.224 & 0.222 \\
\hline
\end{tabular}

Importantly, reducing the step in the number electrons in Eq.4 does not fix the problem of the electric field of charged images unless $\delta N$ is taken at the zero limit. A computational way to take that limit is to compute the density of the neutral system, $\rho\left(\vec{r}, N_{0}\right)$, and several slightly charged systems, $\rho\left(\vec{r}, N_{0}+\delta\right), \rho\left(\vec{r}, N_{0}+2 \delta\right) \ldots$ Then, for every point in the space, a linear interpolation of the density as a function of the number of electrons can be made. The slope of such interpolation is the Fukui function because

$$
\rho(\vec{r}, N)=\rho\left(\vec{r}, N_{0}\right)+\frac{\partial \rho(\vec{r}, N)}{\partial N}\left(N-N_{0}\right)+\cdots
$$

Of course, this approximation will be valid only if the charge of the charged systems is small enough to fall within the linear regime of $\rho$ vs. $N$. This interpolation approach fixes the charged images problem and takes into account the relaxation of inner states. However, it introduces a new difficulty, which is the lack of accuracy of approximate functionals to describe the electron density of systems with fractional number of electrons. It is well known that LDA, all GGA's and most hybrids and long-range corrected functional fail to predict the piece-wise Evs.N function. ${ }^{31}$ All those functionals follow the same trend, which is a convex underestimation of $E$ vs.N. This underestimation results in a delocalization error in the density. That is, the density of systems with fractional number of electrons computed with approximated functionals is less compact than the exact one.

We have written a Python script to evaluate the Fukui function from the interpolation 
scheme of Eq. 10. A copy of it is provided in the supplementary material or upon request.

Table 3 displays the computed $f^{-}$with the interpolation of selected systems with different fractional number of electrons. The $\delta q$ values are chosen to be small enough to fall within the linear behavior of the electron density i.e. close to the neutral system. Three different charges have been computed for each system, with $\delta q$ ranging between -0.30 and -0.05 e, as represented by the interpolation scheme column in the Table. In some cases the neutral system has been included in the interpolation. A linear interpolation point-by-point is carried out and a grid file containing the interpolated values of $f^{-}$is obtained. Condensation is done within the Voronoi polyhedra. The results show that the values of condensed $f^{-}$on the oxygen sites are robust and do not exhibit significant deviations for a given composition: $\mathrm{MgO}$ always shows $0.168, \mathrm{CaO}$ and $\mathrm{SrO}$ oscillate between 0.170 and 0.173 , and $\mathrm{BaO}$ between 0.157 and 0.158 . No significant change is observed by including the neutral density in the interpolation. The results are consistent with the values obtained with finite differences of charged systems (Table1). Although the numbers are smaller than those obtained for the DOS approach, the trend of $\mathrm{BaO}$ values smaller than $\mathrm{MgO}, \mathrm{CaO}$ and $\mathrm{SrO}$ is conserved.

Table 3: $f^{-}$condensed to $\mathrm{O}$ atoms obtained with the interpolation method for the four bulk systems of the alkaline earth metal oxide series

\begin{tabular}{lllll}
\hline interpolation scheme & $\mathrm{MgO}$ & $\mathrm{CaO}$ & $\mathrm{SrO}$ & $\mathrm{BaO}$ \\
\hline$-0.15 /-0.10 /-0.05 / 0.00$ & 0.168 & 0.170 & 0.172 & 0.157 \\
$-0.30 /-0.20 /-0.10 / 0.00$ & 0.168 & 0.172 & 0.171 & 0.157 \\
$-0.15 /-0.10 /-0.05$ & 0.168 & 0.172 & 0.172 & 0.157 \\
$-0.30 /-0.20 /-0.10$ & 0.168 & 0.173 & 0.171 & 0.158 \\
\hline
\end{tabular}

To summarize, the results obtained for the bulk systems show that the different approaches lead to qualitative agreement i.e. bulk $\mathrm{BaO}$ shows smaller $f^{-}$values than $\mathrm{MgO}$, $\mathrm{CaO}$, SrO, irrespective the scheme used. The values obtained are robust as regards the numerical settings within the approaches followed, although the numbers are found smaller when considering charged systems (finite differences and interpolation schemes) than DOS. A remark here is that the values of condensed FF are all normalized to one. This means that if the size of the unit cell is doubled, the values obtained will be halved, since there 
will be twice oxygen sites. A convenient way to express the values could thus be to give the FF value per unit formula, which would apply to systems with the same stoichiometry and allow comparison among them.

\section{Application to surfaces}

The analysis of the different computational schemes to calculate $f^{-}$can be used to illustrate the different reactivity of oxygen sites located at the surface and in bulk positions. It is expected that surface sites are more reactive than bulk sites, due to the low coordination of the former that raises electronic levels. In this section we analyse if such behavior is captured by the Fukui Function $f^{-}$, together with the effect of relaxing the slab symmetrically or not. Figure 1 shows the two sets of slab models used. In the asymmetric slab the two uppermost layers (labelled 1 and 2) have been relaxed, the rest is frozen to the bulk positions, whereas in the symmetric slab the two top and bottom layers are relaxed (labelled 1, 2, 5 and 6), keeping the inner layers (3 and 4) frozen to bulk positions. We have chosen to analyze the interpolation scheme which we define in this work for the first time, and the DOS scheme for comparison purposes.

The $f^{-}$condensed to oxygen atoms has been obtained for the asymmetric slabs of the four oxides. Table 4 summarizes the results for two schemes of interpolation of charged systems, $\delta q=-0.30 /-0.20 /-0.10$ with and without the neutral system. The results are very similar to the interpolation scheme with $\delta q=-0.15 /-0.10 /-0.05(/ 0.00)$, which are compiled in the supplementary material. It can be observed that, in all the systems and interpolation schemes used, the surface oxygen site display the highest values of the condensed Fukui Function, ranging from $0.099-0.084$ for $\mathrm{MgO}$ to $0.128-0.140$ for $\mathrm{SrO}$ in the first interpolation scheme (the second, not considering the neutral system, leads to similar results). The inner layers show lower values, from 0.008 for $\mathrm{BaO}$ to 0.046 for $\mathrm{MgO}$ and $\mathrm{SrO}$. This is a clear indication of the increase in the reactivity of surface sites compared to inner positions. The comparison with the bulk is not straightforward because the condensed $f^{-}$values are 


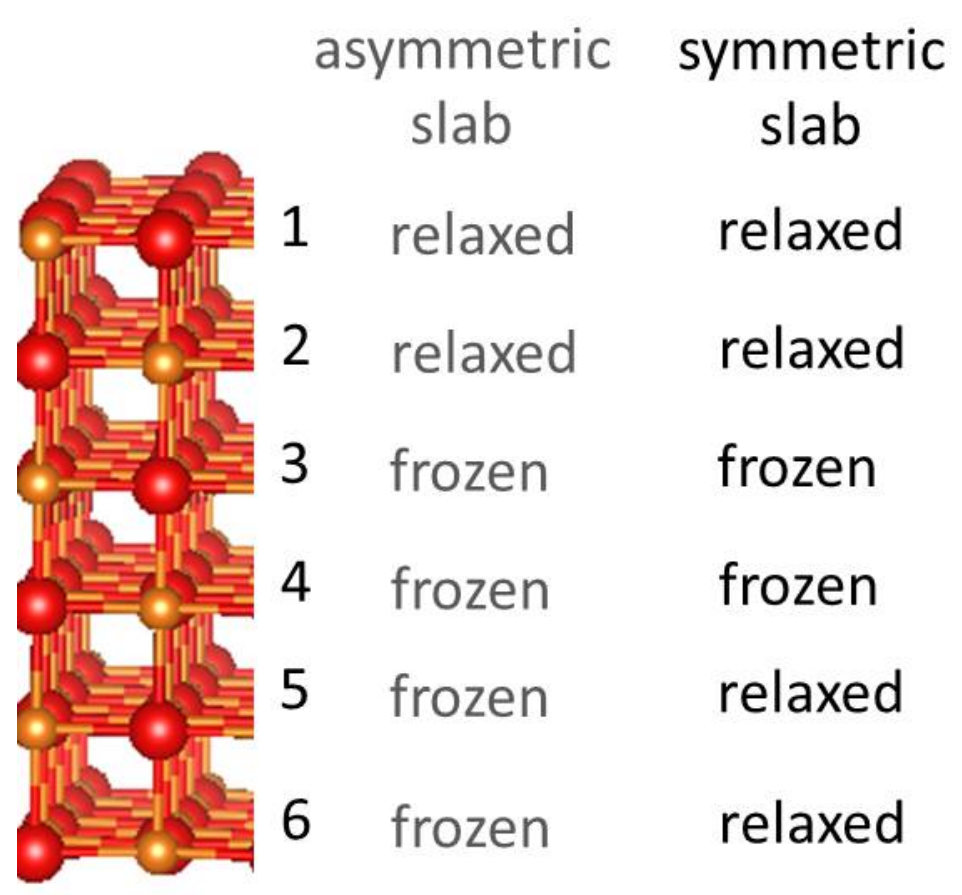

Figure 1: The slab models used in this work to study the surfaces. Layers are labelled with numbers 1-6.

extensive i.e. $f^{-}$always integrates to 1 independently of the number of atoms. In the case of surfaces a distribution of the Fukui among all the positions is observed. The same behavior occurs for the four oxides although different numbers are obtained.

It is interesting to note that structural relaxation of the surfaces leads to a variation in the value of $f^{-}$for all the oxides but for $\mathrm{CaO}$ : oxygen in layer 1 and in layer 6 seem to react in a different manner to the removal of electrons, which is reasonable considering that the electronic states around the Fermi level are affected by the geometrical relaxation. There is not a clear trend within in the oxides, since in $\mathrm{MgO}$ the relaxed $\mathrm{O}$ shows higher values (0.099 relaxed vs 0.084 unrelaxed), $\mathrm{CaO}$ show similar values (0.116 vs 0.115$)$, SrO higher values for the unrelaxed $\mathrm{O}$ (0.128 relaxed vs 0.140 unrelaxed), as $\mathrm{BaO}$ (0.113 relaxed vs 0.130 unrelaxed). The value of $\delta q$ does not influence much the outcome, although in the case of $\mathrm{BaO}$ the $-0.15 /-0.10 /-0.05$ scheme leads to similar values of relaxed and unrelaxed sites (see supplementary material). What is clear is that an accurate relaxation of a surfaces is of uttermost importance to assess its reactivity. Small changes in geometry may not change 
the energy significantly, but they change the position (relative to the Fermi Level) of surface states.

In order to analyze the role of the computational method in the description of the surface oxygen $f^{-}$we have also computed the descriptor with the DOS approach for different $\delta \mu$. Table 5 shows the data obtained for $\mathrm{MgO}$ and $\mathrm{BaO}$ asymmetric and symmetric slabs, with selected values for $\delta \mu$ and interpolation scheme. It can be observed that the two approaches account for the increase in reactivity of the surface sites compared to inner positions. Also, the symmetric slabs reflect the same value for layers 1 and 6 as expected from the relaxation, whereas the unrelaxed sites of layer 6 show lower values than layer 1 in $\mathrm{MgO}$ and higher in $\mathrm{BaO}$, following the trend discussed above.

Interestingly, the calculation of $f^{-}$with the DOS approach is found to be very sensitive to the value of $\delta \mu$ used for the integration in Eq. 9. Figure 2 shows the profile of the condensed FF for the different layers from the data of Table 5. MgO shows a very asymmetric profile for the DOS approach of the asymmetric slab. BaO shows a large difference between layers 1 and 6 with the same approach. The results for symmetric slabs are more homogeneous. In the supplementary material three different ranges are studied and it can be observed that the profile of the Fukui function condensed in the different layers depends on $\delta \mu$. It seems that small integration ranges lead to large variations in the profiles, maybe due to numerical instability in such tiny ranges. Values of $\delta \mu$ of -0.20 and -0.30 lead to results similar to the interpolation approach for the scheme $-0.30 /-0.20 /-0.10$ and could be a good starting point.

In summary, the Fukui function $f^{-}$condensed to oxygen atoms appears to be a good descriptor for the surface reactivity within a given material. The different approaches tested give the same qualitative results: the surface $\mathrm{O}$ atoms are more reactive (the $f^{-}$is larger) than the inner layers atoms. The interpolation of equation 10 is a robust way to obtain $f^{-}$in non charged systems, and is found to be sensitive to the relaxation state of the atom. Attention must be paid to the parameters used to obtain $f^{-}$i.e. the partial charges $\delta q$ in the charged systems (finite differences and interpolation schemes), the $\delta \mu$ range to integrate the 
Table 4: $f^{-}$condensed to $\mathrm{O}$ atoms obtained with the interpolation method for the asymmetric slabs

\begin{tabular}{lcccc}
\hline layer & $\mathrm{MgO}$ & $\mathrm{CaO}$ & $\mathrm{SrO}$ & $\mathrm{BaO}$ \\
\hline$-0.30 /-0.20 /-0.10 / 0.00$ & & & & \\
1 & 0.099 & 0.116 & 0.128 & 0.112 \\
2 & 0.038 & 0.027 & 0.023 & 0.025 \\
3 & 0.035 & 0.036 & 0.046 & 0.023 \\
4 & 0.036 & 0.022 & 0.017 & 0.016 \\
5 & 0.046 & 0.025 & 0.012 & 0.008 \\
6 & 0.084 & 0.115 & 0.140 & 0.129 \\
bulk & 0.168 & 0.172 & 0.171 & 0.157 \\
\hline$-0.30 /-0.20 /-0.10$ & & & & \\
1 & 0.101 & 0.112 & 0.122 & 0.108 \\
2 & 0.038 & 0.027 & 0.028 & 0.030 \\
3 & 0.034 & 0.023 & 0.024 & 0.027 \\
4 & 0.036 & 0.022 & 0.020 & 0.018 \\
5 & 0.046 & 0.025 & 0.013 & 0.008 \\
6 & 0.085 & 0.110 & 0.134 & 0.122 \\
bulk & 0.168 & 0.173 & 0.171 & 0.158 \\
\hline
\end{tabular}

Table 5: comparison of $f^{-}$obtained by integration of the DOS $(\delta \mu=-0.20 \mathrm{eV})$ and the interpolation of $\delta q-0.30 /-0.20 /-0.10$ for asymmetric and symmetric slabs of $\mathrm{MgO}$ and $\mathrm{BaO}$

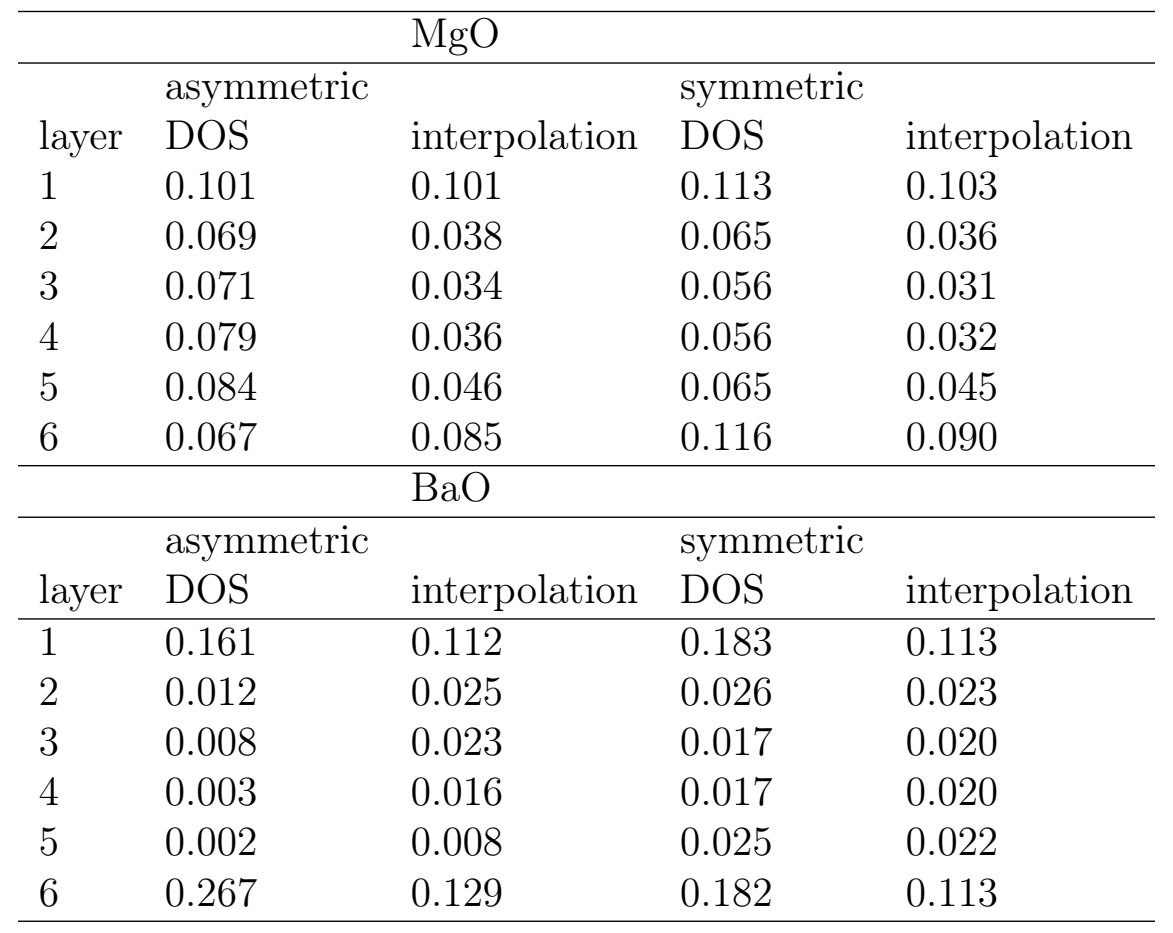



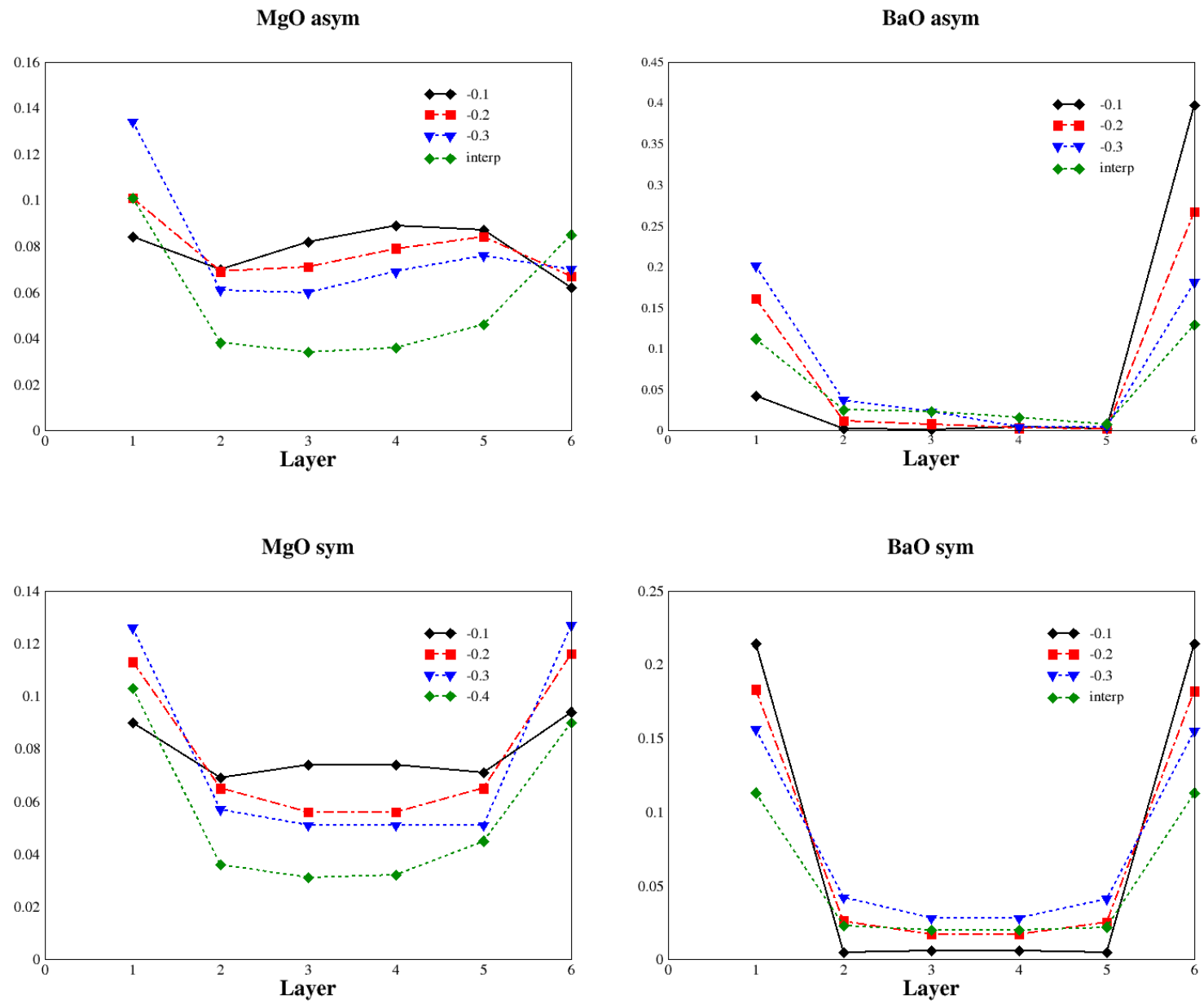

Figure 2: $f^{-}$for the $\mathrm{O}$ sites of the slab layers for $\mathrm{MgO}$ and $\mathrm{BaO}$ systems, asymmetric and symmetric slabs. The following settings are represented: integration of DOS within $-0.10,-0.20$ and $-0.30 \mathrm{eV}$ and the Fermi level, and the interpolation of charged systems for $-0.30 /-0.20 /-0.10|\mathrm{e}|$ 
DOS, which should not take too much small values. Comparison between surfaces and bulk, or between $\mathrm{MgO}-\mathrm{CaO}-\mathrm{SrO}-\mathrm{BaO}$ is not straightforward because the computed $f^{-}$always normalizes to one independently of the nature of the system and the number of atoms in the simulation cell. Comparison of reactivity between systems of the same size could be achieved by using the local softness instead of the Fukui function. As for the matter of extensivity of the condensed Fukui function, an alternative is to standardize that, whenever possible, its

value be reported multiplied by the number of unit formulas in the cell. For instance, the values of the Fukui of the $\mathrm{O}$ in the bulks of $\mathrm{MgO}$ and $\mathrm{BaO}$ in Table 3 and the central layers (3 and 4 ) in Table 4 become comparable if multiplied by 2 and 12 respectively.

This means that it does not take into account the total number of electrons in the system, nor the size or the nature of the elements. Providing the FF per unit formula, and considering the global softness, may improve the description and expand the limits of use of Fukui functions.

\section{Conclusions}

In this work we have presented methods to compute the Fukui function $f^{-}$in periodic boundary conditions within the density functional theory framework, and apply them to alkaline earth oxides bulk and surface models. The approaches to calculate $f^{-}$are based on i) finite differences of charge density for integer and non-integer $\delta q$ partially charged cells ii) the integration of the local density of states for a $\delta \mu$ interval and iii) the interpolation of the charged density obtained for partially charged systems. All the methods provide robust qualitative results; however special attention must be given to the choice of the $\delta q$ and $\delta \mu$ to obtain a stable description. The interpolation method appears as an appropriate way to account for the relaxation of inner states and to improve the finite differences description when using charged systems. It is particularly sensitive to the effect of geometry relaxation in slab models and seems thus well suited to study solid state reactivity. 


\section{Supporting Information Available}

Computed properties of bulk systems, geometrical parameters of surfaces, FF computed for different approaches and MO systems, geometrical structures of asymetric slab models (POSCAR format), python script to interpolate charges density files and examples of application

\section{Acknowledgement}

This work was financed by: i) FONDECYT through project No 1181121, ii) CONICYT, REDES 190102 iii) Centers Of Excellence With Basal-Conicyt Financing, Grant FB0807. Powered@NLHPC: This research was partially supported by the supercomputing infrastructure of the NLHPC (ECM-02). M.L.C. thanks to the Internal Research project of Universidad Finis Terrae (2018). This work was performed using HPC resources from GENCICINES/IDRIS (Grant 2019- x2019082131) and the LCT cluster.Dr. B. Diawara is warmly akcnowledged for the Modelview visualisation program.

\section{References}

(1) Klopman, G. Chemical reactivity and the concept of charge and frontier-controlled reactions. Journal of the American Chemical Society 1968, 90, 223-234.

(2) Salem, L. Intermolecular orbital theory of the interaction between conjugated systems. I. General theory. Journal of the American Chemical Society 1968, 90, 543-552.

(3) Fukui, K. Role of frontier orbitals in chemical reactions. Science 1982, 218, 747-754.

(4) Parr, R. G.; Donnelly, R. A.; Levy, M.; Palke, W. E. Electronegativity: the density functional viewpoint. Journal of Chemical Physics 1978, 68, 3801-3807. 
(5) Chermette, H. Chemical reactivity indexes in density functional theory. Journal of Computational Chemistry 1999, 20, 129-154.

(6) Liu, S. B. Conceptual Density Functional Theory and Some Recent Developments. Acta Physico-Chimica Sinica 2009, 25, 590-600.

(7) Gazquez, J. Perspectives On Density Functional Theory Of Chemical Reactivity. Journal of the Mexican Chemical Society 2008, 52, 3-10.

(8) Fuentealba, P.; Cardenas, C. In Chemical Modelling: Volume 11; Michael Springborg, J.-O. J., Ed.; The Royal Society of Chemistry, 2015; Vol. 11; pp 151-174.

(9) Geerlings, P.; De Proft, F. Conceptual DFT: the chemical relevance of higher response functions. Physical Chemistry Chemical Physics 2008, 10, 3028-3042.

(10) Geerlings, P.; De Proft, F.; Langenaeker, W. Conceptual density functional theory. Chemical Reviews 2003, 103, 1793-1873.

(11) Miranda-Quintana, R. A.; Heidar-Zadeh, F.; Ayers, P. W. Elementary Derivation of the " $\Delta \mu$ Big Is Good" Rule. J Phys Chem Lett 2018, 9, 4344-4348.

(12) Miranda-Quintana, R. A.; Ayers, P. W. The " $|\Delta \mu|$ big is good" rule, the maximum hardness, and minimum electrophilicity principles. Theoretical Chemistry Accounts 2019, 138.

(13) Parr, R. G. Companions in the search. International Journal of Quantum Chemistry 1994, 49, 739-770.

(14) Cardenas, C.; Ayers, P. W.; Cedillo, A. Reactivity indicators for degenerate states in the density-functional theoretic chemical reactivity theory. The Journal of Chemical Physics 2011, 134, 174103-13. 
(15) Bultinck, P.; Cardenas, C.; Fuentealba, P.; Johnson, P. A.; Ayers, P. W. Atomic Charges and the Electrostatic Potential Are Ill-Defined in Degenerate Ground States. Journal of Chemical Theory and Computation 2013, 9, 4779-4788.

(16) Bultinck, P.; Cardenas, C.; Fuentealba, P.; Johnson, P. A.; Ayers, P. W. How to Compute the Fukui Matrix and Function for Systems with (Quasi-)Degenerate States. Journal of Chemical Theory and Computation 2013, 10, 202-210.

(17) Bultinck, P.; Jayatilaka, D.; Cardenas, C. A problematic issue for atoms in molecules: Impact of (quasi-)degenerate states on Quantum Theory Atoms in Molecules and Hirshfeld-I properties. Computational and Theoretical Chemistry 2015, 1053, 106-111.

(18) Perdew, J. P.; Parr, R. G.; Levy, M.; Balduz, J., Jose L. Density-functional theory for fractional particle number: derivative discontinuities of the energy. Physical Review Letters 1982, 49, 1691-1694.

(19) Echegaray, E.; Rabi, S.; Cardenas, C.; Zadeh, F. H.; Rabi, N.; Lee, S.; Anderson, J. S.; Toro-Labbe, A.; Ayers, P. W. In pursuit of negative Fukui functions: molecules with very small band gaps. Journal of Molecular Modeling 2014, 20, 1-7.

(20) Fuentealba, P.; Cardenas, C.; Pino-Rios, R.; Tiznado, W. Applications of Topological Methods in Molecular Chemistry; Springer International Publishing, 2016; pp 227-241.

(21) Kresse, G.; Hafner, J. Abinitio Molecular-Dynamics for Liquid-Metals. Physical Review $B$ 1993, 47, 558-561.

(22) Kresse, G.; Furthmuller, J. Efficiency of ab-initio total energy calculations for metals and semiconductors using a plane-wave basis set. Computational Materials Science 1996, $6,15-50$.

(23) Kresse, G.; Furthmüller, J. Efficient iterative schemes for ab initio total-energy calculations using a plane-wave basis set. Physical review B 1996, 54, 11169. 
(24) Blochl, P. E. Projector Augmented-Wave Method. Physical Review B 1994, 50, 1795317979 .

(25) Perdew, J. P.; Burke, K.; Ernzerhof, M. Generalized Gradient Approximation Made Simple. Physical Review Letters 1996, 77, 3865-3868.

(26) Pack, J. D.; Monkhorst, H. J. Special Points for Brillouin-Zone Integrations - Reply. Physical Review B 1977, 16, 1748-1749.

(27) Logsdail, A. J.; Mora-Fonz, D.; Scanlon, D. O.; Catlow, C. R. A.; Sokol, A. A. Structural, energetic and electronic properties of (100) surfaces for alkaline earth metal oxides as calculated with hybrid density functional theory. Surface Science 2015, 642, 58-65.

(28) Cardenas, C.; De Proft, F.; Chamorro, E.; Fuentealba, P.; Geerlings, P. Theoretical study of the surface reactivity of alkaline earth oxides: Local density of states evaluation of the local softness. Journal of Chemical Physics 2008, 128, 034708.

(29) Calatayud, M.; Ruppert, A.; Weckhuysen, B. M. Theoretical Study on the Role of Surface Basicity and Lewis Acidity on the Etherification of Glycerol over Alkaline Earth Metal Oxides. Chemistry: A European Journal 2009, 15, 10864-10870.

(30) Tang, W.; Sanville, E.; Henkelman, G. A grid-based Bader analysis algorithm without lattice bias. Journal of Physics: Condensed Matter 2009, 21, 084204.

(31) Cohen, A. J.; Mori-Sanchez, P.; Yang, W. T. Insights into current limitations of density functional theory. Science 2008, 321, 792-794.

(32) Sablon, N.; Proft, F. D.; Geerlings, P. Molecular orbital-averaged Fukui function for the reactivity description of alkaline earth metal oxide clusters. Journal of chemical theory and computation 2009, 5, 1245-1253.

(33) Cerón, M. L.; Calatayud, M. Application of dual descriptor to understand the activity 
of $\mathrm{Cu} / \mathrm{ZrO}_{2}$ catalysts in the water gas shift reaction. Journal of molecular modeling 2017, 23, 34 .

(34) Calatayud, M.; Tielens, F.; De Proft, F. Reactivity of gas-phase, crystal and supported $\mathrm{V}_{2} \mathrm{O}_{5}$ systems studied using density functional theory based reactivity indices. Chemical Physics Letters 2008, 456, 59-63.

(35) Pino-Rios, R.; Yañez, O.; Inostroza, D.; Ruiz, L.; Cardenas, C.; Fuentealba, P.; Tiznado, W. Proposal of a simple and effective local reactivity descriptor through a topological analysis of an orbital-weighted fukui function. Journal of Computational Chemistry 2017, 38, 481-488.

(36) Cárdenas, C.; Muñoz, M.; Contreras, J.; Ayers, P. W.; Gómez, T.; Fuentealba, P. Understanding chemical reactivity in extended systems: exploring models of chemical softness in carbon nanotubes. Acta Physico-Chimica Sinica 2018, 34, 631-638.

(37) Cedillo, A.; Cárdenas, C. Reactivity of Carbon Molecular Clusters from a Hückel-Type Model. The Journal of Physical Chemistry A 2019, 123, 8696-8701. 


\section{Graphical TOC Entry}

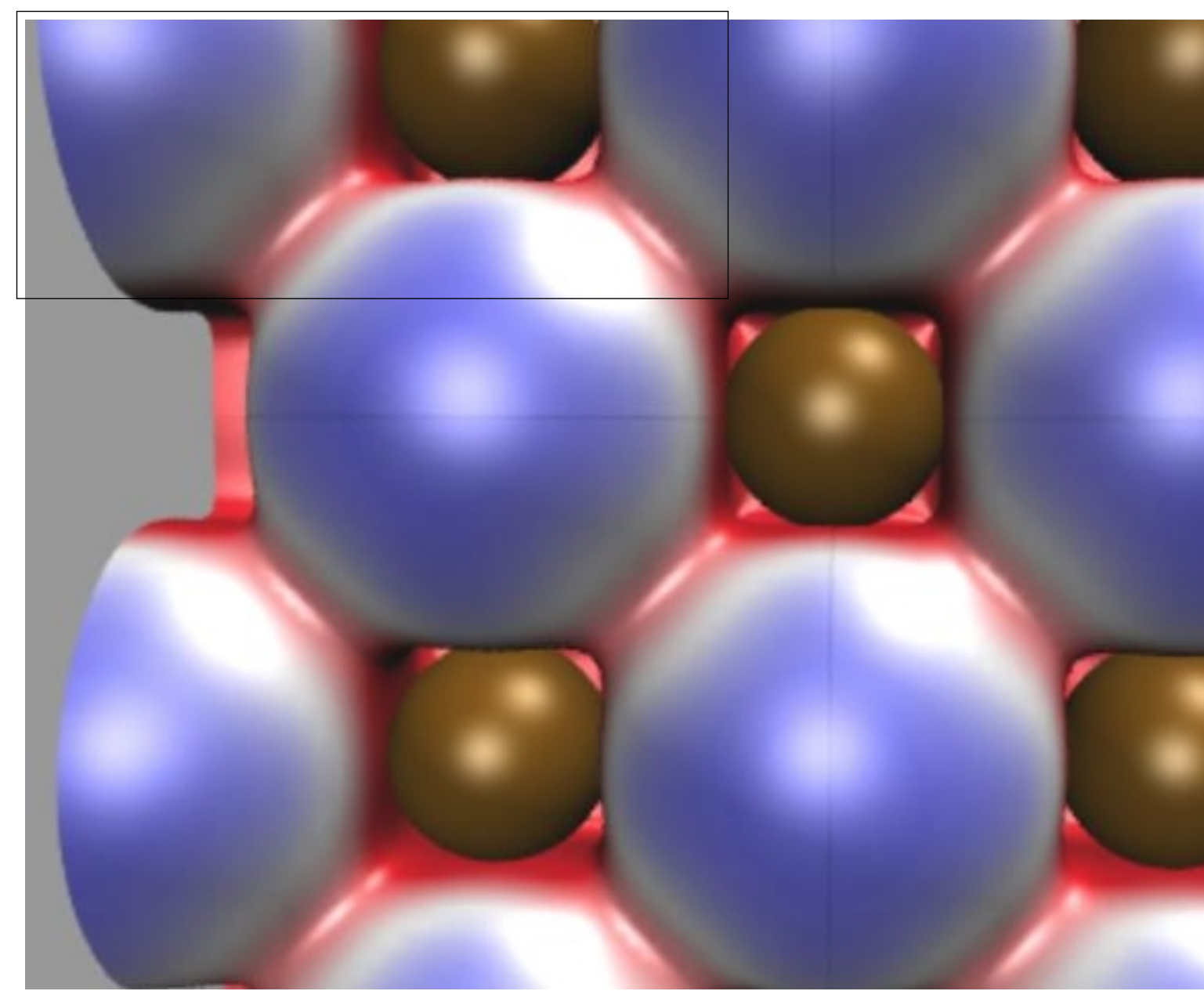

Fukui function shows oxidable sites in alkaline-earth oxides bulk and surfaces 